\title{
Proposal of a Hybrid QAM-MPPM Technique for Optical Communications Systems
}

\author{
Haitham S. Khallaf* and Hossam M. H. Shalaby, Senior Member, IEEE \\ Egypt-Japan University of Science and Technology (E-JUST), Alexandria, Egypt \\ *e-mail: Haitham.khallaf@ejust.edu.eg,eng.h.khallaf@gmail.com
}

\begin{abstract}
M-ary quadrature amplitude modulation (QAM) is a class of non-constant envelope scheme that can achieve high bandwidth efficiency under an average signal power constraint. In this paper, a hybrid M-ary quadrature amplitude modulation multi-pulse pulse-position modulation (hybrid QAM-MPPM) technique is proposed for optical communications systems. Both transmitter and receiver block diagrams, which utilize intensity modulation/direct detection (IM/DD) scheme, are introduced. The proposed system is analyzed theoretically and its bit-error rate (BER) performance is derived and investigated. Our results reveal that, under the conditions of fixed data rates, bandwidth, and energy per bit, the proposed technique outperforms both traditional QAM and MPPM sub-carrier modulation techniques.
\end{abstract}

Keywords: direct detection (DD), hybrid modulation, intensity-modulation (IM), multi-pulse pulse-position modulation (MPPM), quadrature amplitude modulation (QAM).

\section{INTRODUCTION}

M-ary quadrature amplitude modulation (QAM) technique is widely used in modern communications systems when high throughput values are required under limited bandwidth conditions. That is, it is an effective way of increasing the spectral efficiency in communications systems. On the other hand, multi-pulse pulse-position modulation (MPPM) has been considered as an effective power efficient technique in optical communication systems [1]. In this paper, we propose a new hybrid modulation technique to make advantages of both power efficient MPPM and bandwidth efficient QAM schemes. Furthermore, we focus on IM/DD technology to keep the system simple and cheap.

The idea of mixing different modulation techniques has been recently proposed in many literatures. Liu et al. have introduced a new class of optical modulation formats based on combining m-PPM or m-FSK with additional polarization and/or phase modulation [2]. Also, they have presented the principle, implementation, and performance of high-sensitivity modulation format based on the combined use of polarization-division multiplexed quadrature phase-shift keying (PDM-QPSK, or PQ) and M-ary pulse-position modulation (m-PPM) [3]. Selmy et al. have proposed hybrid BPSK-modified MPPM, which outperforms both traditional BPSK and MPPM techniques [4]. Also, they have proposed a new hybrid modulation technique based on both quadrature phase shift keying (QPSK) modulation and modified-MPPM technique. Their proposed scheme achieves much less bit error rate levels than that of traditional QPSK modulation [5]. Khallaf et al. have proposed a hybrid orthogonal frequency-division multiplexing pulse-position modulation (OFDM-PPM) technique for turbulent free-space optical communications systems [6].

In this paper, we have introduce a simple and power efficient technique. Intensity modulation/direct detection scheme has been used in the proposed technique so that there is no need for optical oscillator. Also, mixing power efficient MPPM with bandwidth efficient M-ary QAM results into a new scheme which is more bandwidth efficient if compared to traditional MPPM and more power efficient if compared to traditional M-QAM.

The remainder of this paper is organized as follows. In Section 2, the block diagrams of both transmitter and receiver of the proposed system are presented, along with its mathematical description. In Section 3, the proposed system is analyzed theoretically and its bit-error rate (BER) performance is derived. Section 4 is devoted for the numerical evaluation of the BER, where the proposed technique is compared to traditional MPPM and QAM subcarrier modulation. Finally, the conclusion is given in Section 5.

\section{PROPOSED SYSTEM MODEL}

\subsection{Frame Structure and Transmitter Side}

In MPPM techniques, the symbol duration $T$ is divided into $N$ time slots and has optical power in w $<N$ time slots only. MPPM technique transmits $\log _{2}\left(\begin{array}{l}N \\ w\end{array}\right)$ bits per frame. In the proposed technique, instead of transmitting constant optical power during the signal slot, we send QAM symbol as shown in the time frame structure of hybrid QAM-MPPM technique shown in Fig. 1. In hybrid QAM-MPPM technique, number of bits per frame is $\log _{2}\left(\begin{array}{l}N \\ w\end{array}\right)+{ }^{\prime} \log _{2} M_{q}$. where $M_{q}$ is the number of modulation levels of M-QAM. 


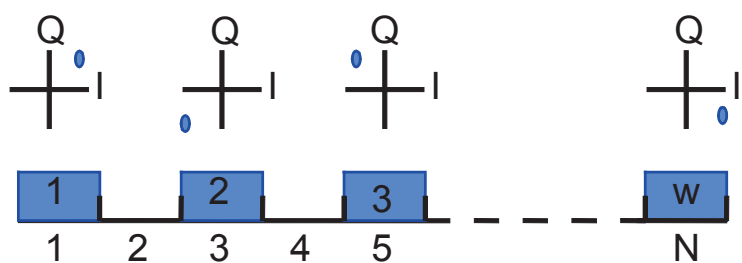

Figure 1. Frame structure of a hybrid QAM-MPPM modulation scheme.

The optical power output from a laser diode is proportional to the modulating QAM signal [7]:

$$
P(t)=\frac{N p}{w} \sum_{i=0}^{N-1}\left[1+M C_{i}(t)\right] \operatorname{rect}\left(t-\frac{i T}{N}\right)
$$

where $p$ is the average transmitted optical power, $M$ is the modulation index, $C_{i}(t)$ and rect $(t)$ are defined as following:

$$
\begin{array}{lr}
C_{i}(t)=\left\{\begin{array}{lr}
S_{Q A M}(t) & \text { for signal time slot } \\
0 & \text { for non signal time slot }
\end{array}\right. \\
\operatorname{rect}(t)=\left\{\begin{array}{lr}
1 & 0 \leq t<T / N \\
0 & \text { otherwise }
\end{array}\right.
\end{array}
$$

The QAM signal $S_{Q A M}(t)$ is defined as following:

$$
S_{Q A M}(t)=A_{I} \cos \left(2 \pi f_{c} t\right)+A_{Q} \cos \left(2 \pi f_{c} t\right)
$$

where $A_{I}$ and $A_{Q}$ are the in-phase and quadrature components of the QAM symbol, respectively, and $f_{c}$ is the electrical carrier frequency.

The basic configuration of the transmitter of the proposed scheme is shown in Fig. 2. The input data are divided into frames each of them consists of $\log _{2}\left(\begin{array}{l}N \\ w\end{array}\right)+w \log _{2} M_{q}$ bits. The first $\log _{2}\left(\begin{array}{l}N \\ w\end{array}\right)$ bits are used by the transmitter DSP to control the locations of the $w$ signal slots. The remainder of the frame bits, $w \log _{2} M_{q}$ bits, are encoded in $w$ QAM symbols.

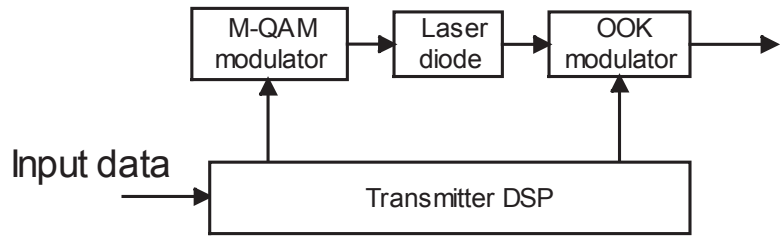

Figure 2. Block diagram of a hybrid QAM-MPPM transmitter.

\subsection{Receiver Side}

The basic configuration of the receiver of the proposed scheme is shown in Fig. 3. At the receiver side of the system, the photodiode converts the received optical intensity variations into corresponding variations in the electrical domain. The received electrical signal $y(t)$ can be written as:

$$
y(t)=R \frac{N}{w} p(1+M C(t))+n(t)
$$

where $R$ is the responsivity of the photodiode and $n$ is a signal-independent zero-mean white Gaussian noise with variance $\sigma_{n}^{2}$.

$$
\begin{aligned}
r_{I} & =R \frac{N}{w} p M A_{I}+n_{1}(t) \\
r_{Q} & =R \frac{N}{w} p M A_{Q}+n_{2}(t)
\end{aligned}
$$

$r_{I}$ and $r_{Q}$ are independent Gaussian random variables with mean values $R \frac{N}{w} M p A_{I}$ and $R \frac{N}{w} M p A_{Q}$, respectively. Their variance is $\sigma_{n}^{2}$. Summation of the square of these independent Gaussian random variables will be used to demodulate the MPPM symbol where the receiver will choose slots that have the highest $w$ energy to be considered as the signal slots. The result of this summation will be a new random variable that has non-central and central chi-square distribution with two degree of freedom in case of signal and non-signal slots, respectively. After demodulating the MPPM symbol, the QAM symbol, which is delayed by one frame duration, will be demodulated and the whole symbol will be reconstructed. 


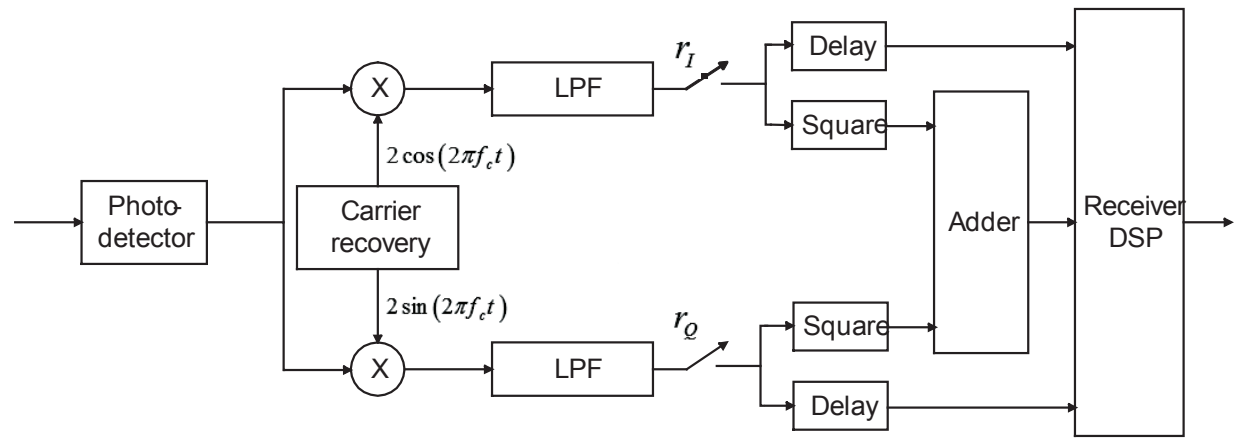

Figure 3. Block diagram of a hybrid QAM-MPPM receiver.

\section{BIT-ERROR RATE ANALYSIS}

The BER of the proposed scheme is the average of the BERs of both QAM and MPPM [2] as given in Eq. (5), where $B E R_{Q A M}$ is the bit-error rate of traditional QAM and $S E R_{M P P M}$ is the symbol-error rates of the traditional MPPM. The first term in (5) accounts for bit error rate that occurs to the group of $\log _{2}\left(\begin{array}{l}N \\ w\end{array}\right)$ bits which is transmitted using MPPM so this group has the bit error rate of MPPM. The second term of the equation accounts for bit error rate of the remaining ${ }^{\prime} \log _{2} M_{q}$ bits. The second term consists of two parts, the first one considers the case when all signal slots are correctly decoded and the second considers the case of wrong decoding. Eq. (6) presents the BER of the traditional Gray coded QAM modulation technique $B E R_{Q A M}$ [8], where $k=\log _{2} M_{q}$. The $S E R_{M P P M}$ is introduced in Eq. (7) [9], where $p_{\min }$ denotes the minimum average power in signal slots, $p_{0}()$ and $p_{1}()$ denote the average power probabilities of non-signal and signal slots, respectively. Also, $P_{0}()$ and $P_{1}()$ denote their cumulative distributions. The average power of the proposed system will have chi-square distribution as mentioned in previous section.

$$
\begin{aligned}
& B E R=\frac{\log _{2}\left(\begin{array}{l}
N \\
w
\end{array}\right)}{\log _{2}\left(\begin{array}{l}
N \\
w
\end{array}\right)+w \log _{2} M_{q}} B E R_{M P P M}+\frac{w \log _{2} M_{q}}{\log _{2}\left(\begin{array}{l}
N \\
w
\end{array}\right)+w \log _{2} M_{q}}\left[\left(1-S E R_{M P P M}\right) B E R_{Q A M}+\frac{S E R_{M P P M}}{2}\right] \\
& B E R_{Q A M} \begin{cases}=\frac{4}{\log _{2} M_{q}}\left(1-\frac{1}{\sqrt{M_{q}}}\right) \sum_{i=1}^{\frac{\sqrt{M_{q}}}{2}} Q\left((2 i-1) \sqrt{\frac{3 S N R}{M_{q}-1}}\right), & \text { when } k \text { is even } \\
<\frac{4}{\log _{2} M_{q}} Q\left((2 i-1) \sqrt{\frac{3 S N R}{M_{q}-1}}\right), & \text { when } k \text { is odd }\end{cases} \\
& S E R_{M P P M}=\sum_{l=1}^{N-w} \sum_{m=1}^{w} \int_{0}^{\infty}\left(\begin{array}{c}
w \\
m
\end{array}\right)\left(\begin{array}{c}
N-w \\
l
\end{array}\right) p_{1}\left(p_{\text {min }}\right)^{m}\left(1-P_{1}\left(p_{\text {min }}\right)\right)^{w-m}\left(P_{0}\left(p_{\text {min }}\right)\right)^{N-w-l} \\
& \text { * }\left[\left(1-P_{0}\left(p_{\min }\right)\right)^{l}+p_{0}\left(p_{\min }\right)^{l}\left(1-\frac{1}{\left(\begin{array}{c}
l+m \\
m
\end{array}\right)}\right)\right] d p_{\min }
\end{aligned}
$$

\section{NUMERICAL RESULTS}

In this section we use expressions shown in the last section to investigate the performance of the proposed scheme. In these comparisons, we choose the parameters for system so that they have the same data rates, same average energy and same bandwidth. Figure 4 shows the average BERs for both hybrid QAM-MPPM and traditional QAM systems versus average transmitted optical power. As shown in the Fig. 4, the proposed hybrid QAM-MPPM scheme outperforms the traditional QAM and is more power efficient. The proposed system saves about $1 \mathrm{~dB}$ in average power at BER of $10^{-3}$. The reason for this improvement is the increase in the available average power for a QAM symbol of the proposed system. The average power of the QAM symbol in hybrid QAM-MPPM scheme is $\frac{N}{w}$ times that of the traditional QAM symbol (in order to keep the data rates of both schemes equal as well as keep equal average energy). This increases the signal to noise ratio (SNR) seen by the QAM symbol of proposed scheme.

Figure 5 shows the average BERs for both proposed technique and traditional MPPM technique versus average transmitted optical power. Proposed system outperform the traditional system by $4 \mathrm{~dB}$ at BER $10^{-3}$. In proposed system 2 pulses only are transmitted during 12 time slots while traditional MPPM will transmit 5 pulses during the same number of time slots in order to have the same number of bits per symbol as the new 
technique. So the hybrid QAM-MPPM system will have higher SNR comparing the traditional MPPM in order to conserve the condition of equal average energy.

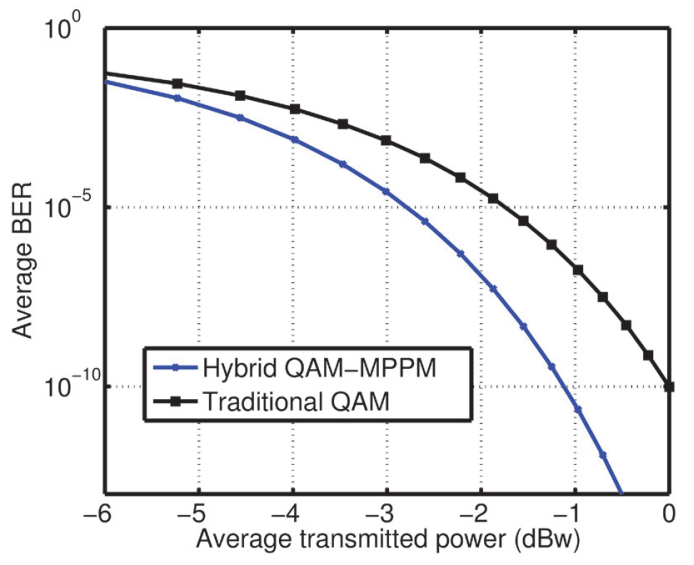

Figure 4.Average bit-error rates for both hybrid QAM$M P P M$ (with $N=4, w=2$, and $M_{q}=8$ ) and traditional $Q A M\left(\right.$ with $\left.M_{q}=4\right)$ versus average transmitted optical power in $d B w$.

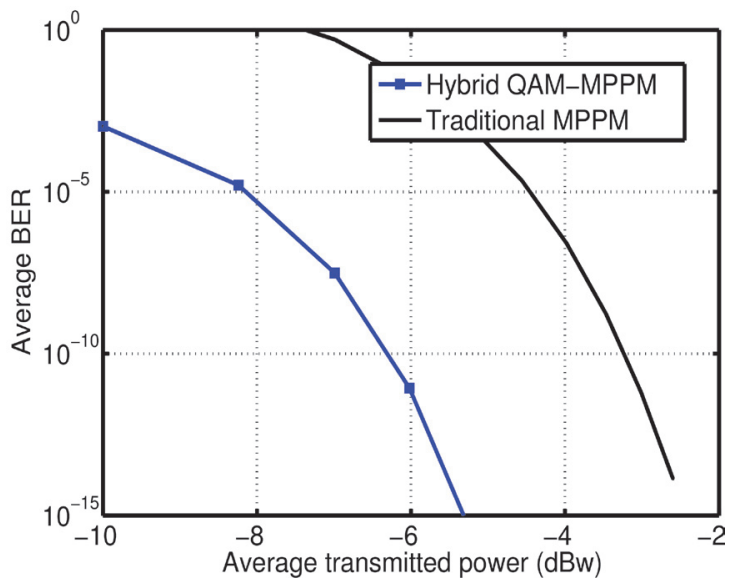

Figure 5.Average bit-error rates for both hybrid QAMMPPM (with $N=12, w=2$, and $M_{q}=4$ ) and traditional MPPM (with $N=12$ and $w=5$ ) versus average transmitted optical power in $\mathrm{dBw}$.

\section{CONCLUSION}

A hybrid QAM-MPPM modulation technique has been proposed as a power efficient modulation technique. Mathematical model, transmitter and receiver of the proposed scheme have been presented. The bit error rate (BER) of the proposed system has been derived with the effect of the signal independent noise taken into consideration. The BER performance of the proposed hybrid QAM-MPPM scheme has been compared to that of traditional QAM and MPPM schemes under the conditions of same transmitted data rates, same bandwidth and, same average energy. Our results reveal that the hybrid QAM-MPPM system outperforms the traditional techniques and is more power efficient.

\section{ACKNOWLEDGMENT}

The authors would like to thank Egyptian Ministry of Higher Education (MoHE) and Egypt-Japan University of Science and Technology (E-JUST) for their support.

\section{REFERENCES}

[1] H. Sugiyama and K. Nosu, "MPPM: a method for improving the band utilization efficiency in optical PPM,” J. Lightw. Technol., vol. 7, no. 3, pp. 465-472, Mar. 1989.

[2] X. Liu, S. Chandrasekhar, T. Wood, R. Tkach, P. Winzer, E. Burrows, and A. Chraplyvy, "M-ary pulseposition modulation and frequency-shift keying with additional polarization/phase modulation for highsensitivity optical transmission," Opt. Express, vol. 19, no 26, pp. B868-B881, 2011.

[3] X. Liu, T. H. Wood, R. W. Tkach, and S. Chandrasekhar, "Demonstration of record sensitivities in optically preamplified receivers by combining PDM-QPSK and M-ary pulse-position modulation," J. Lightw. Technol, vol. 30, no. 4, pp. 406-413, Feb. 2012.

[4] H. Selmy, H. M. H. Shalaby, and Z. Kawasaki., "Proposal and performance evaluation of a hybrid BPSKmodified mppm technique for optical fiber communications systems," J. Lightw. Technol, 2013.

[5] H. Selmy, H. M. Shalaby, and Z. Kawasaki., "Performance enhancement of qpsk modulation using hybrid QPSK-modified MPPM in optical fiber communications," in Proc. International Japan-Egypt Conference on Electronics, Communications and Computers (JEC-ECC), 2013, pp. 196-198.

[6] H. S. Khallaf, H. M. H. Shalaby, and Z. Kawasaki., "Proposal of a hybrid OFDM-PPM technique for free space optical communications systems," in Proc. IPC, 2013.

[7] A. Bekkali, C. Ben Naila, K. Kazaura, K. Wakamori, and M. Matsumoto, "Transmission analysis of OFDM-based wireless services over turbulent radio-on-FSO links modeled by gamma-gamma distribution," IEEE Photonics Journal, vol. 2, no. 3, pp. 510-520, 2010.

[8] F. Xiong, Digital Modulation Techniques, 2nd ed. ARTECH HOUSE, INC., 2006.

[9] A. E. Morra, H. S. Khallaf, H. M. H. Shalaby, and Z. Kawasaki., "Performance analysis of both shot- and thermal-noise limited multipulse ppm receivers in gamma-gamma atmospheric channels," J. Lightw. Technol., vol. 31, no. 19, pp. 3142-3150, Oct 2013. 